\title{
Les proximités, entre contrainte et libération de l'action : le cas d'EADS
}

\section{Damien Talbot}

\section{(2) OpenEdition \\ 12 Journals}

Édition électronique

URL : http://journals.openedition.org/ei/939

DOI : 10.4000/ei.939

ISSN : 2553-1891

\section{Éditeur}

Association Économie et Institutions

\section{Édition imprimée}

Date de publication : 1 décembre 2005

Pagination : 153-180

ISSN : 1775-2329

\section{Référence électronique}

Damien Talbot, «Les proximités, entre contrainte et libération de l'action : le cas d'EADS », Économie et institutions [En ligne], 6-7 | 2005, mis en ligne le 31 janvier 2013, consulté le 19 avril 2019. URL : http:// journals.openedition.org/ei/939; DOI : 10.4000/ei.939 


\title{
Les proximités, entre contrainte et libération de l'action : le cas d'EADS 1
}

\author{
Damien TALBOT
}

\section{Introduction}

L'objet de cet article est de procéder à un travail de définition et de qualification des formes de proximité 2 . Derrière cette notion, se sont regroupés quelques chercheurs qui souhaitent aborder le rôle de l'espace dans la coordination, en lui octroyant une dimension sociale fondatrice. Vient en effet se superposer à cet espace physique des actions collectives qui transforment le contexte purement physique en un espace construit par les hommes. Ce n'est pas uniquement à l'espace physique, simple contexte fonctionnel, auquel nous faisons référence ici, mais aussi à l'idée que les individus s'en font, la représentation mentale de celui-ci pouvant être partagée et devenir ainsi une référence commune. L'espace représenté devient un médiateur entre les hommes, favorisant leurs relations.

Si l'acteur est situé dans un espace géographique, il l'est tout autant dans un espace social. Grâce aux technologies de communications et aux déplacements, il est possible d'être "proche " de quelqu'un de géographiquement éloigné (Rallet et Torre, 2004), à l'instar d'établissements répartis sur différents sites et appartenant à un même groupe, qui partagent dans ce cas une forme non spatiale de proximité. Ainsi, au-delà de la seule dimension spatiale, cette notion est destinée à rendre compte de la dynamique des réseaux d'interaction qui apparaissent comme des construits issus des représentations et des comportements acteurs. Finalement, la proximité se déploie dans diverses dimensions: une dimension spatiale, on parle alors de proximité géographique, et une dimension relationnelle qui renvoie à l'action collective. Les deux formes de proximités peuvent d'ailleurs se combiner, à l'exemple du cas particulier du territoire dans lequel se déploie une action collective localisée.

Si la définition de la proximité géographique fait l'objet d'un large consensus au sein des chercheurs qui composent le groupe "Dynamiques de Proximité ", il n'en est pas de même du versant relationnel de ce concept. Un premier courant qualifié

\footnotetext{
1 European Aeronautic Defence and Space Company.

2 Pour les publications les plus récentes sur ce point, le lecteur peut se référer à Gilly et Torre (2000), Dupuy et Burmeister (2003), Pecqueur et Zimmermann (2004) ou encore Torre et Filippi (2005).
} 
d'interactionniste $^{3}$ se limite à deux formes de proximité (géographique, organisée), tandis qu'un second d'inspiration institutionnaliste (au sens large du terme) décline la proximité en trois composantes (géographique, institutionnelle, organisationnelle) (Gilly et Lung, 2004). Le premier courant définit la proximité organisée comme la capacité qu'offre une organisation de faire interagir ses membres. Cette capacité est le résultat d'une logique d'appartenance qui traduit le fait que les membres d'une organisation interagissent effectivement et d'une logique de similitude qui exprime la communauté de croyances et de savoirs que lie les participants (Rallet et Torre, 2004). Si la logique de similitude a vocation à capter la dimension institutionnelle de la proximité, cette définition présente l'inconvénient à nos yeux de ne pas épuiser, sans toutefois l'occulter, l'influence des objets sociaux collectifs que sont les institutions. Plus précisément, nous décelons deux limites dans cette définition. Une première renvoie à la fonction des institutions. La logique de similitude assimile en effet les phénomènes institutionnels à des phénomènes d'abord cognitifs, sans rendre compte du rôle fondamentalement politique des institutions. Celles-ci sont tout autant des prescriptions sociales régulatrices de conflits que des repères. Une seconde limite relève plus d'un problème de méthode. En n'intégrant pas d'emblée les institutions, le risque est important de sous estimer leur rôle, de ne pas comprendre le sens d'un ordre social réduit à un ensemble d'interactions interindividuelles désincarnées des habitudes collectives qui les sous-tendent. Si l'on admet que l'action humaine n'est pas que fonctionnelle (survie, reproduction, etc.), alors son sens est à trouver dans le poids du collectif, de l'histoire, des apprentissages, des pouvoirs, des relations de confiance, des valeurs, etc. L'enjeu n'est donc pas de faire appel ponctuellement au " contexte institutionnel " comme un facteur "sociologique " parmi d'autres facteurs, de réduire l'institution à un signal qui autorise la construction d'anticipations. De même que les chercheurs du groupe se sont rassemblés autour de la nécessité d'endogénéiser l'espace dans la théorie économique, nous plaidons pour une endogénéisation des institutions : il s'agit de débuter l'analyse des comportements par l'affirmation du rôle des institutions sur ceux-ci, sans déterminisme aucun puisque l'intentionnalité des agents est affirmée, leur volonté s'exerçant bien mais dans un champ des possibilités institutionnellement définis. Autrement dit, nous affirmons avec

3 Pour une discussion sur les différences entre les approches individualiste, holiste et interactionniste, $c f$. par exemple Grossetti et Fillipi (2004). Ces auteurs expliquent notamment que le point commun des travaux interactionnistes est de rejeter la figure de l'agent représentatif au profit d'un agent qui tient compte dans son action du comportement des autres agents avec lesquels il entretient des liens directs.

154 Economie et Institutions $-n^{\circ} 6$ et $7-1^{\text {er }}$ et $2^{\text {e }}$ semestres 2005 
Hodgson (2000) que les institutions ne sont pas extérieures aux individus qui les portent. Pour dépasser ces deux limites nous distinguons alors, au côté de la proximité géographique, une proximité institutionnelle et une proximité organisationnelle.

Afin de définir ces deux dernières dimensions de la proximité, nous mobiliserons la distinction réalisée par Commons (1934) entre institution et organisation. La proximité organisationnelle sera ainsi entendue comme une forme particulière de proximité institutionnelle, tandis que nous soulignerons le rôle ambigu de la proximité géographique. Une fois ces définitions posées, nous examinerons comment un groupe (EADS) récemment crée (en 2000) construit de telles proximités institutionnelle et organisationnelle entre ses diverses unités, appartenant il y a encore peu de temps à trois firmes distinctes, Aerospatiale-Matra pour la partie française, Dasa (Daimler Chrysler) pour la partie allemande et Casa pour la partie espagnole. L'analyse des proximités construites par EADS dans ses relations verticales permettra d'étendre notre illustration au cas d'une proximité géographique ${ }^{4}$.

\section{Une définition institutionnaliste des proximités}

Dans cette première partie, nous définissons tour à tour les trois formes de proximité en cherchant à montrer la pertinence de la mobilisation d'une perspective institutionnaliste. Notamment, nous expliquons en quoi les proximités apparaissent comme des contraintes et des ressources pour l'action.

\subsection{La proximité institutionnelle : contrainte et ressource pour l'action}

Selon Pecqueur et Zimmermann, la notion de proximité "correspond à une capacité d'agents qui la partagent à se coordonner" (Pecqueur et Zimmermann, 2004, p. 25). Dans ses déclinaisons spatiales et non spatiales, elle cherche à rendre compte des conditions nécessaires à la coordination des agents. Elle est tout à la fois une contrainte (limites spatiales, limites culturelles à la coopération, externalités négatives) et un potentiel qui peut faciliter des coordinations, d'abord ponctuelles, puis parfois plus régulières et plus intenses. Ces relations peu à peu renouvelées génèrent progressivement des avantages accessibles aux participants, comme autant de ressources pour l'action.

4 Une part importante de ces éléments provient des résultats d'un contrat de recherche auquel nous avons contribué (Bécue et al., 2005). 
Il est frappant de constater la similitude entre cette définition de la proximité et celle de l'institution donnée par Commons qui y voit, lui aussi, une contrainte et une ressource pour l'action en réponse à l'incertitude inhérente à celle-ci. La réponse humaine à cette incertitude consiste notamment à répéter certains comportements dont les conséquences futures sont plus ou moins connues, ayant été déjà observées. La répétition présente en outre l'avantage de réduire la recherche d'information et le besoin d'analyse de la situation qui précédent toute action. Ainsi, face à un monde incertain et complexe, les individus se créent des habitudes rendant leurs anticipations plus sûres. Leurs propres habitudes individuelles dérivent des habitudes collectives (comme les coutumes) et de règles plus structurées (comme les lois) qui exercent une pression sociale : les individus auront alors tendance à s'y conformer.

Ces comportements répétitifs lorsqu'ils sont collectifs et stabilisés sont alors qualifiés d'institution par certains institutionnalistes américains comme Commons (1931)5. Si l'on adopte une perspective pragmatique qui rejette le dualisme cartésien entre corps et esprit, l'action apparaît comme indissociable du niveau cognitif qui lui donne sens. Inversement, la fonction de la pensée est de créer des "habitudes d'action" (Grinberg, 2001). La compréhension de l'action humaine suppose alors d'articuler les représentations et les comportements : l'action pour Commons est une opinion en action (Bazzoli et Dutraive, 2002), cette opinion individuelle étant pour partie façonnée par les opinions collectives, par l'histoire de l'acteur et par ses représentations du futur.

Dit autrement, les institutions apparaissent comme orientées vers une fin. En ce sens elles sont artificielles, profondément humaines, encastrées dans une social-historicité selon les termes de Jullien (2004). Comme les actions ne sont ni neutres ni objectives, mais instituées, elles sont construites en fonction des finalités et doivent d'être examinées en référence à ces mêmes fins. Commons introduit le concept de futurité (futurity) dans son analyse pour révéler l'importance de la question des finalités. Gislain (2002) explique que, selon Commons, les hommes vivent dans le futur et agissent dans le présent. Leurs actions sont fonction de leurs hypothèses habituelles concernant les conséquences futures de leurs actions présentes. La futurité se définit alors comme une représentation d'un but à atteindre dans le futur par les actions présentes. C'est un devenir commun plus ou moins sécurisé et structuré collectivement, composé de significations et de règles de

5 Bazzoli et Dutraive (2002) expliquent que certains institutionnalistes américains, comme Veblen, insistent plus sur la dimension cognitive des institutions en développant la notion d'habitudes de pensée tandis que d'autres, comme Commons, s'attachent à approfondir leur aspect structurel. Le refus pragmatique de la dichotomie pensée/action réunit ces auteurs. 
conduites permettant à chacun de s'inscrire dans le projet commun (Gislain, 2003). Cette futurité est perpétuellement actualisée par les acteurs. Elle est le sujet de conflits, de processus de négociation, de compromis temporaires, de construction d'accords transitoires pour continuer à vivre ensemble dans un projet durable. Dés lors, Gislain définit les institutions, suivant en cela Commons (1934), comme " des actions collectives qui construisent et contrôlent, sans les déterminer totalement, les actions individuelles " (2003, p. 14), actions collectives inscrites dans une futurité. Les institutions sont donc à la fois des contraintes et des ressources pour l'action individuelle (Corei, 1995). L'action commune devient alors possible car l'action individuelle, à la fois contrainte et libérée, est instituée.

Le terme de proximité institutionnelle désigne le fait pour un groupe d'individus de partager et de se conformer à un ensemble d'institutions. De la même façon que l'on partage un espace physique construit, les agents partagent un espace social composé d'institutions dont l'adoption, qui résulte tout autant de la volonté de l'individu que des rapports de pouvoir existants, conduit à formation d'un monde commun. Cette proximité institutionnelle contraint et libère à la fois l'action de l'individu.

Elle contraint l'action individuelle car :

- elle marque les limites d'un espace possible de coordination au-delà duquel l'action collective est impossible. Autrement dit, les agents sont situés dans un faisceau d'interrelations qui conditionnent leurs activités productives, commerciales, d'innovation, etc. (Pecqueur et Zimmermann, 2004). Stipuler l'agent comme situé permet de restreindre son potentiel d'interaction à son environnement de proximités, de définir son voisinage social, de délimiter l'aire d'influence des institutions ;

- au sein de cet espace possible de coordination, l'action est aussi contrainte dans sa forme. Se conformer à des institutions revient à adopter des comportements attendus : elles peuvent alors se comprendre comme des prescriptions sociales. En d'autres termes, ces actions collectives orientées vers des fins sont une expression des rapports de pouvoir existant entre les individus. Concrètement, un agent peut légitimement exercer une domination en créant et en imposant par exemple ses propres règles et objectifs à autrui (pensons au cas de l'autorité dirigeante et de ses salariés).

L'action est libérée par la réduction de l'incertitude qui lui est inhérente. Cette réduction a une triple origine: l'homogénéisation des comportements circonscrits et prescrits que nous venons d'évoquer, la subjectivité des institutions, la régulation des conflits. 
En effet :

- les institutions, comme autant de repères, établissent des modèles: en bornant les comportements, elles rendent ces derniers plus semblables et donc plus prévisibles même si des problèmes d'interprétation peuvent se poser. Ainsi la proximité institutionnelle favorise un pronostic adéquat du comportement stabilisé d'autrui ;

- les institutions partagées, orientées vers une fin, sont porteuses de sens, de valeurs, voire d'engagements réduisant l'incertitude. Il s'agit là d'une vision positive de leur finalité, les acteurs les mobilisant non seulement pour satisfaire leurs intérêts individuels ou collectifs, mais aussi parce qu'elles leur semblent justes ;

- une action contrainte dans son étendue et orientée dans sa forme, des comportements donc plus semblables auxquels s'ajoutent le fait que les institutions, comme catégories, fonctionnent comme des filtres n'autorisant le traitement que des informations considérées comme acceptables par les acteurs qui n'entrent pas ou faiblement en contradiction avec leurs valeurs, sont autant de facteurs de réduction temporaire des conflits. Le passage de divers compromis fondant l'action collective devient alors possible. Ces compromis portent notamment sur la compréhension des problèmes existants et sur la façon de les résoudre en vue d'atteindre un but commun.

Ainsi, dans un monde moins incertain du fait de l'existence d'institutions, des relations délimitées et orientées deviennent possibles entre de multiples individus. Il en est ainsi de l'organisation qui constitue, au côté des coutumes, des habitudes, des règles juridiques ou morales, etc., une manifestation concrète des institutions.

\subsection{La proximité organisationnelle : une forme particulière de proximité institutionnelle}

La proximité organisationnelle "lie les agents participant à une activité finalisée dans le cadre d'une structure particulière. (...) La proximité organisationnelle se déploie à l'intérieur des organisations (firmes, établissements, etc.) et, le cas échéant, entre organisations liées par un rapport de dépendance ou d'interdépendance économique ou financière (entre sociétés membres d'un groupe industriel ou financier, au sein d'un réseau, etc.)" (Kirat et Lung, 1995, p. 213). Ainsi, l'individu qui intègre une organisation partage de fait une proximité organisationnelle avec l'ensemble des membres. Suivant la distinction opérée par Commons (1934) entre institution et 
organisation, nous comprenons la proximité organisationnelle comme une forme de proximité institutionnelle. Il s'agit toujours de partager un espace social qui prend cette fois-ci une forme concrète observable par tous (à la différence par exemple d'une coutume), celle d'une organisation comprise comme un atome de sociétés humaines (Grinberg, 2001).

Une organisation renvoie à des comportements collectifs orientés vers un but commun explicite qui résulte d'un compromis, encadrés par des règles de fonctionnement et par un "gouvernement " propre qui exerce le pouvoir. Dans une perspective institutionnaliste, l'organisation, à l'instar des entreprises, devient un lieu de production et d'activation de règles qui assurent une coordination de nature cognitive, répondant à la problématique de l'efficacité des actions, et politique, répondant à la problématique de la conformité et de la légitimité des mêmes actions réalisées par des acteurs hétérogènes. La coordination cognitive se fonde sur le caractère homogénéisant d'institutions porteuses de sens (action individuelle libérée) limitant l'espace possible de coordination (action individuelle contrainte). La coordination politique est rendue possible parce que les institutions sont des prescriptions sociales (action individuelle contrainte) régulatrices des conflits (action individuelle libérée).

Sur le plan cognitif, et selon Bazzoli et Dutraive (2002), les règles d'action que chacun élabore face à l'incertitude ont une fonction cognitive indispensable de construction des représentations à travers des expériences empiriques quotidiennes, tout en constituant des guides pour l'action. Autrement dit, il s'agit par les règles de réduire l'incertitude et de sécuriser les anticipations. Ces règles ne sont pas incompatibles avec la rationalité. Elles sont même une condition nécessaire à son exercice, en économisant les capacités cognitives pour les activités routinières qui seront dès lors utilisées de façon opportunes. "Dans cette perspective, la rationalité n'est pas associée à l'optimisation ni à la délibération consciente au cas par cas; elle correspond à la capacité humaine à concentrer l'activité intelligente sur un nombre limité de facteurs spécifiques pour aboutir à un résultat satisfaisant et repose sur la routinisation des processus cognitifs qui dispensent les individus d'une complète attention et délibération." (Bazzoli et Dutraive, 2002, p. 27). Autrement dit, la routinisation des actions, loin de s'opposer à l'efficacité, la conditionne.

La notion de routine développée par les approches évolutionnistes de la firme illustre bien la nécessité de cette coordination cognitive. Pour utiliser une terminologie évolutionniste, les organisations comme les firmes apparaissent comme des producteurs de routines visant à l'efficacité de l'action. Les routines sont des "modèles d'interactions qui constituent des solutions efficaces 
à des problèmes particuliers" (Dosi et al., 1990, p. 243). Elles se composent de séquences codées déterminant un enchaînement de décisions et de comportements (Ménard, 1994) décrivant ce que doivent être les pratiques individuelles. Ce sont des habitudes, des institutions qui présentent un caractère très concret et qui stockent un savoir-faire tacite se maintenant dans le temps. Ces routines vont dans le sens d'une réduction de l'incertitude et de sécurisation des anticipations: par leurs caractères mécaniques et automatiques, elles suspendent en effet l'incertitude liée à l'action de l'autre, et apparaissent comme une stabilisation de l'interprétation des règles car elles "(...) sont un mode de résolution pragmatique d'un problème auquel les règles dont une réponse théorique (...)" (Reynaud, 2001, p. 64). Les routines et les règles sont autant de ressources cognitives qui vont faire l'objet d'un apprentissage de la part des membres de l'organisation. Il existe alors un processus d'accumulation de telles ressources en vue de réaliser des tâches de manière efficace et qui correspond à la construction d'une mémoire organisationnelle.

Toutefois, l'institutionnalisme se démarque des compréhensions évolutionnistes des organisations, en ce sens que celles-ci, comme formes institutionnelles, ne doivent pas être réduites à une seule fonction de coordination cognitive. Si l'on s'en tient à l'exemple de la firme, la division interne du travail relève à la fois d'une nécessité cognitive de donner un contenu à des tâches qu'il faut rendre complémentaires, mais aussi d'une nécessité politique d'articuler les actions d'acteurs aux statuts et aux intérêts différents (direction, salariés, syndicat, etc.) comme réponse aux questions de pouvoir et d'appropriation. Sur un plan politique, Bazzoli et Dutraive (2002) en analysant la pensée de Commons caractérisent les organisations, au regard des institutions, de la façon suivante :

- pour exister, une organisation doit avoir une constitution juridique qui renvoie aux buts de l'institution créée et exprime les relations de pouvoir et d'autorité. Elle repose sur des règles externes, des règles de droit qui fondent l'existence légale de l'organisation en déléguant un pouvoir normateur et de mobilisation des ressources à ses représentants, et sur des règles internes, qui organisent l'exercice du pouvoir, définissent les termes de l'appartenance à l'organisation et qui permettent la réalisation de l'activité. L'organisation productrice de règles incarne ici matériellement l'institution. De même, la proximité organisationnelle est la matérialisation de la proximité institutionnelle ;

- une organisation, si elle est une structure, est aussi un processus qui permet de produire la volonté, la motivation à 
participer des membres. Cette motivation est le résultat du désir de chaque individu d'être approuvé par les autres et de l'existence d'une futurité commune aux acteurs, d'une représentation partagée d'objectifs à atteindre grâce aux actions présentes. La question de l'intentionnalité est au cœur de la définition de l'organisation, et plus généralement de l'action collective. Ainsi, partager une proximité organisationnelle est le résultat d'une volonté, d'un acte conscient des acteurs qui y voient une façon de satisfaire leurs intérêts individuels et collectifs dans le champ des possibles défini par les institutions ;

- une organisation est une institution si elle perdure dans le temps, malgré le changement de ses membres. Cette longévité, qui autorise la constitution d'une mémoire, est bien entendu fonction de la capacité l'organisation à faire évoluer ses règles pour s'adapter. L'organisation comme institution est bien une entité évolutive.

Nous définissons alors les organisations comme des institutions dotées, d'une part, d'une mémoire composée de règles et de routines comprises comme autant de ressources cognitives mobilisées pour la mise en cohérence des actions individuelles et pour atteindre efficacement les objectifs fixés (coordination cognitive) et, d'autre part, de structures de pouvoir qui ont en charge cette mise en cohérence en incitant/veillant à la conformité de ces actions aux règles produites, ce qui permet leur légitimation (coordination politique). La proximité organisationnelle consistera alors pour des acteurs a priori hétérogènes à intégrer la communauté cognitive, c'est-à-dire à accéder à la mémoire de l'organisation composée de ressources cognitives, et à intégrer la communauté politique, c'est-àdire à s'insérer dans la structure de pouvoir. Elle est bien une forme particulière de proximité institutionnelle qui consiste, rappelons-le, à partager des institutions, ici des règles et des routines, et à s'y conformer, ici en intégrant une structure de pouvoir.

\subsection{La proximité géographique : un rôle ambigu}

Il nous reste à définir la forme la plus intuitive de la proximité, dite géographique. Il ne s'agit pas d'une simple proximité physique, notion qui repose sur une conception naturelle de l'espace. La proximité géographique se fonde au contraire sur une vision artificialiste de celui-ci : de la même façon que l'action individuelle est instituée, l'espace physique peut l'être aussi. 


\subsubsection{L'espace institué : un construit social porteur d'une identité}

Nous l'avons déjà évoqué en introduction, l'espace géographique ne doit pas uniquement s'entendre comme un contexte purement physique doté d'attributs matériels au sein duquel se déroulent des relations économiques. Cet espace possède une dimension sociale fondatrice qui permet de le saisir plus comme une construction active de relations que comme un "réceptacle neutre et uniforme" des stratégies des acteurs (Dupuy et Gilly, 1996). Nous nous situons dans une "conception d'un espace physique, construit, travaillé, modelé, partagé par les hommes. (...) Les espaces construits sont des transformations de l'espace physique préexistant, conservant comme fondement le cadre matériel des interactions et des échanges " (Grossetti, 1997, p. 3). L'espace est un objet de mémoire, composée du nom de cet espace, de ses limites physiques, de son histoire, de son patrimoine, de modes de vie, des coordinations précédentes réussies ou échouées, etc. Cet objet de mémoire constitue dès lors un référent cognitif, l'espace intervenant dans le processus de construction (destruction) des identités dans le sens où il est une composante essentielle du rapport aux autres (Pecqueur, 1997) puisque les acteurs locaux se le représentent et le font exister au regard des autres (Laganier, Villalba et Zuindeau, 2004). Bref, l'espace est institué, ce qui signifie que l'existence d'une proximité géographique entre acteurs agissant en commun n'est pas neutre, que le fait de partager un même lieu peut contraindre et libérer l'action commune.

1.3.2. La proximité géographique comme une contrainte: une source de conflits

N'oublions pas que la proximité géographique qui lie certains acteurs est d'abord subie et peut se transformer en une contrainte pour l'action collective, tant on voit mal les membres d'une association, d'un syndicat professionnel ou encore des élus se délocaliser pour se soustraire à un voisinage indésirable. Etre voisin peut signifier s'ignorer, d'autant que les technologies de communications offrent dorénavant la possibilité d'être présent ici et ailleurs (Rallet et Torre, 2004). Cela peut aussi signifier se détester car, si la proximité géographique autorise une meilleure connaissance de l'autre du fait du partage d'une identité minimale, cette connaissance peut conduire tout autant les acteurs à la confiance qu'à la défiance, en fonction de la mémoire des coordinations réussies ou échouées. De façon intermédiaire, le voisinage peut aussi engendrer la méfiance. Ainsi, il peut exister des conflits latents résultant de relations passées. C'est notamment le cas lorsque l'objectif de l'action collective est de permettre une 
utilisation raisonnée d'une ressource consommée conjointement entre les usagers d'un même lieu (pensons à l'eau), après une période entachée par des conflits de voisinage au sujet de problèmes de répartition, de pollutions diffuses etc., qui auraient affectés la ressource. Ici, la coordination politique est peu ou mal assurée, tant la régulation des conflits est défaillante du fait d'intérêts opposés. La littérature sur les conflits d'usage de l'espace (voir dans ce numéro les articles de Caron et Torre et de Jeanneaux et Kirat; $c f$. aussi Caron et Torre, 2004 ; Kirat et Lefranc, 2004) cite nombre d'exemples d'inégalités liées à la situation topographique des acteurs (un usager subit la pollution de l'eau provenant d'un autre usager situé en amont). La proximité géographique peut donc être constitutive d'inégalités créant des rapports de pouvoir entre les acteurs. Ce n'est pas pour autant que toutes les interactions deviennent définitivement impossibles, car les acteurs qui n'ont pas la capacité de se relocaliser ailleurs doivent faire face à cette externalité négative de proximité, selon les termes de Rallet et Torre (2004). Cet état de fait constitue à lui seul une forte incitation au compromis, et plus généralement à la coordination en mobilisant la dimension positive des ressources cognitives communes, c'est-à-dire le lien social identitaire, obligés que sont les acteurs de s'entendre in fine. Ici, la coordination cognitive pallie en partie aux difficultés de coordination politique.

1.3.3. La proximité géographique libératrice de l'action commune: identité et structuration matérielle de l'espace

La proximité géographique aborde la question des conditions objectives de localisation (Pecqueur et Zimmermann, 2004) et traduit la distance géographique entre deux entités, pondérée par les moyens de transport et par le jugement des individus sur la nature d'une telle distance (Rallet et Torre, 2004). Il ne s'agit pas d'affirmer que le partage d'un même espace géographique induit nécessairement des relations entre acteurs, mais que la proximité géographique peut les faciliter voire les structurer et cela de deux façons complémentaires.

Premièrement, le partage d'un même espace facilite la réalisation concrète de la coordination. Nous renvoyons ici à l'idée classique selon laquelle l'espace physique est structuré par des infrastructures de transport et de communication, constituant un cadre matériel favorisant la circulation des informations, des biens physiques et des individus. En particulier, des acteurs ayant pour objectif la production de connaissances vont profiter d'une localisation commune dans un tel espace structuré pour satisfaire leur besoin, au moins temporaire, de face à face. 
Deuxièmement, et sur la base de la conception élargie d'un espace dorénavant institué, nous soulignons que le partage d'une identité facilite les échanges et catalyse les interactions sociales (Vant, 1998) en réduisant l'incertitude inhérente à l'action et en sécurisant les anticipations. Ce lien social peut même se révéler fondateur d'une confiance entre les acteurs locaux et plus généralement de ressources cognitives spécifiques qui, une fois combinées, aident à la résolution de problèmes ( $c f$. l'article de Colletis et Pecqueur, dans ce numéro $\left.{ }^{6}\right)$. Ces ressources localisées sont toujours des résultats fragiles et transitoires, issus des coordinations antérieures de nature à la fois privées et professionnelles. On insiste ici sur la dimension historique de la relation, largement inscrite dans la notion de proximité : l'histoire des liens compte.

$\mathrm{Au}$ final, nous avons défini la proximité institutionnelle comme le fait pour des acteurs de partager et de se conformer à des institutions. La proximité organisationnelle est entendue comme une forme particulière de proximité institutionnelle, dans laquelle les coordinations de nature cognitive et politique conduisent au partage de règles et de routines vecteurs des connaissances et à l'intégration d'une structure de pouvoir. L'analyse en terme de contrainte versus libération a permis de souligner le rôle ambigu de la proximité géographique, qui contraint l'action collective tout autant qu'elle la facilite. Ces trois formes de proximités constituent les trois piliers de l'action collective, sans qu'il soit pour autant nécessaire de les mobiliser toutes à la fois. En particulier, les proximités organisationnelle et géographique peuvent jouer un rôle important dans un type de coordination, organisée et/ou localisée, et occuper une place plus réduite dans un autre, non structurée et/ou aspatiale. Par contre, la proximité institutionnelle apparaît comme une condition nécessaire à toute coordination, étant entendu qu'elle peut varier en intensité : une compréhension mutuelle minimale est en effet nécessaire à toute forme d'action collective, même la plus simple. Muni de cet outil analytique, il est alors possible de procéder à un travail de qualification de diverses formes d'actions collectives. Dans certains cas, à l'exemple du groupe européen du secteur aérospatial EADS, les proximités institutionnelle et organisationnelle suffisent à rendre compte de l'organisation interne. Dans d'autres situations, à l'instar des relations verticales entre EADS et ses soustraitants, la proximité géographique compte.

6 Ces auteurs y définissent les ressources comme un potentiel latent de facteurs à exploiter, à organiser ou à révéler. Elles sont spécifiques lorsqu'elles ne peuvent être transférées.

164 Economie et Institutions $-n^{\circ} 6$ et $7-1^{\text {er }}$ et $2^{\text {e }}$ semestres 2005 


\section{Une illustration des formes de proximité : EADS, organisation interne et relations verticales}

L'exemple d'un groupe industriel comme EADS peut illustrer nos définitions des proximités. Sur le plan politique, il se compose de liens hiérarchiques entre la société mère, constituant le centre de décision, et ses unités (filiales, sociétés contrôlées minoritairement, etc.). Ces structures, qui découlent des propriétés juridiques, sont les vecteurs des relations financières et patrimoniales autorisant plus ou moins d'autonomies financière et stratégique aux unités qui se conforment aux règles édictées par la tête de groupe. Cette dernière régule et contrôle au moins partiellement les relations internes, entre les unités, et externes, entre les unités et son environnement (fournisseurs, clients, financeurs, etc.). Cette régulation et ce contrôle permettent une gestion des conflits stabilisant les anticipations. Il est alors possible de procéder à la définition d'une stratégie, c'est-à-dire d'une futurité, censée provoquer l'adhésion des membres aux projets collectifs. En outre, la société mère a pour fonction de gérer l'allocation des capitaux entre plusieurs activités. Ce rôle d'allocation est l'expression du pouvoir de l'équipe dirigeante, elle-même soumise au contrôle des actionnaires qui orientent la stratégie globale du groupe. Sur le plan cognitif, vont se nouer des rapports de production, qui ne sont plus nécessairement verticaux dans le sens où ils peuvent concerner directement des unités entre elles. Ces rapports résultent des contraintes de la division cognitive du travail au sein du groupe. Cette coordination suppose des mécanismes d'apprentissage ayant pour but la création et l'assimilation de règles et de routines. En outre, les outils développés en interne pour permettre une coordination cognitive sont simultanément appliqués aux relations de sous-traitance tandis que la coordination politique consiste par exemple en une hiérarchisation des fournisseurs. Ces relations verticales se fondent ponctuellement sur une proximité géographique, le cas toulousain en constituant un parfait exemple.

Pour rendre compte précisément des proximités qui lient la tête de groupe à ses unités et le même groupe à ses sous-traitants, nous distinguons deux périodes qui ponctuent l'histoire de l'industrie aéronautique, principal secteur d'activité d'EADS: la première, qui va du début du $\mathrm{XX}^{\mathrm{e}}$ siècle au milieu des années soixante-dix, voit les relations entre les acteurs du secteur régies par un ensemble d'institutions partagées que Muller (1988) a qualifié de logique d'arsenal. Depuis une quarantaine d'années, cette dernière est progressivement remise en cause au profit d'une autre logique de proximité, dite logique de marché (cf. Talbot (2000) pour une analyse de cette dynamique institutionnelle). Cela ne signifie pas pour autant que la logique d'arsenal a totalement disparu. Si EADS est 
l'aboutissement organisationnel concret de l'affirmation de la logique de marché, il n'en reste pas moins que la proximité organisationnelle qui lie les différentes unités d'EADS est aussi le résultat de la persistance de certaines habitudes de comportements qui relèvent d'une logique d'arsenal. Autrement dit, on ne peut pas décrire la coordination politique et la coordination cognitive à l'œuvre dans EADS et entre EADS et ses preneurs d'ordres sans faire référence aux deux logiques.

\subsection{La proximité organisationnelle au sein d'EADS : la coordination politique}

La coordination politique d'EADS présente la particularité de reposer sur une structure bicéphale, conduisant à la désignation de deux présidents, tout en intégrant des Etats dans son actionnariat. Il apparaît en outre que la proximité organisationnelle n'est pas isomorphe, certaines unités disposant de plus ou moins d'autonomie décisionnelle ou étant plus ou moins bien intégrées.

2.1.1. La proximité organisationnelle comme une forme de proximité institutionnelle: des Etats actionnaires et une structure bicéphale résultant de la logique d'arsenal

Saisir la dimension politique de la coordination du groupe EADS suppose l'examen de son actionnariat, relativement atypique puisqu'il rassemble des acteurs publics et privés, qui plus est de nationalités différentes, dans un secteur traditionnellement peu ouvert aux participations étrangères. Le français Lagardère, l'allemand Daimler Chrysler (tous les deux liés par un pacte jusqu'en 2006), les Etats français et espagnols sont les principaux actionnaires de la nouvelle entité7. La présence de ce dernier provient $\mathrm{du}$ fait qu'Aerospatiale était initialement un groupe public jusqu'à sa privatisation qui résulte de la fusion avec Matra Hautes Technologies, l'ensemble intégrant EADS en 2000.

Ainsi, deux acteurs publics participent à la définition de la stratégie du groupe européen. L'Etat français conserve en outre le contrôle des unités d'EADS qui ont en charge la réalisation des missiles balistiques constitutifs de la force de dissuasion nucléaire française. Autre particularité, la présidence du groupe est bicéphale, co-dirigé par un français et un allemand. A l'échelon inférieur, les cadres dirigeants d'origine française sont deux fois plus nombreux

7 En 2004, l'actionnariat d'EADS se compose de la façon suivante: Dasa $32,87 \%$; actionnariat flottant $30,16 \%$; Sogeade $(50 \%$ Lagardère $50 \%$, Etat français 50\%) 30,13\% ; Sepi (Etat espagnol) 5,51\% ; salariés 0,79\% ; Etat français $0,06 \%$.

166 Economie et Institutions $-n^{\circ} 6$ et $7-1^{\text {er }}$ et $2^{\text {e }}$ semestres 2005 
que leurs collègues allemands8. Ce partage du pouvoir est le résultat d'un compromis entre trois coalitions nationales d'acteurs, les Allemands, les Français et les Espagnols. Ce compromis fragile, toujours transitoire, est périodiquement remis en cause9.

Ces règles internes de coordination sont atypiques de l'institution du capitalisme qu'est le groupe, qui adopte traditionnellement une structure du pouvoir strictement hiérarchique conduisant à la désignation d'un seul individu à sa tête. Il faut voir dans cette exception une traduction organisationnelle concrète de la logique d'arsenal qui perdure dans l'industrie aéronautique européenne malgré une profonde remise en cause entamée dans les années soixante-dix. Les acteurs de l'industrie aéronautique sont porteurs dans les années soixante de plusieurs représentations et appliquent des règles de coordination dont le partage est constitutif d'une telle proximité institutionnelle. Ainsi, un avion efficient est un avion performant techniquement (à l'exemple $\mathrm{du}$ Concorde), l'aspect technologique étant prédominant, tandis que les nécessités commerciales sont reléguées au second plan. L'Etat est l'acteur central de cette industrie pour au moins deux ensembles de raisons. Premièrement, l'aéronautique est un secteur dit de "frontière technologique" dans lequel tout pays industrialisé se doit de figurer. Dès lors, l'Etat définit une politique technologique afin de conserver, voire de développer, un niveau technique auquel les seules forces d'un marché trop étroit ne peuvent conduire. De plus, ce secteur provoque des retombées bénéfiques à l'ensemble de l'industrie nationale et concourre au prestige national. Deuxièmement, le caractère militaire d'une partie de l'industrie aéronautique conduit l'ensemble de cette dernière à être placé sous tutelle étatique. Finalement, dans une logique d'arsenal, l'industrie aéronautique apparaît comme un outil au service de la préservation de la souveraineté nationale: la présence d'Etats dans l'actionnariat d'EADS et le système de co-présidence franco-allemande ont vocation à permettre à chaque partenaire de peser sur les décisions, d'orienter la stratégie du groupe dans le sens de la préservation de leurs intérêts nationaux jugés essentiels.

L'existence du groupe EADS intégrant les industries nationales résulte de l'affirmation progressive à partir des années

8 En 2002, sur 177 cadres dirigeants du groupe, on compte 100 Français pour 50 Allemands (La Tribune, 14 novembre 2002).

9 Il suffit pour s'en convaincre d'examiner les luttes d'influences qui ont débuté au premier semestre 2005 pour le renouvellement de l'équipe dirigeante. Les actionnaires allemands estiment que leurs homologues français tentent d'abandonner la structure bicéphale d'EADS pour une présidence unique française. Quelques mois auparavant, les Espagnols ont réclamé un partage plus en leur faveur des responsabilités et une part actionnariale plus importante. 
soixante-dix d'un autre ensemble d'institutions, qualifié ici de logique de marché. Dorénavant, selon une telle proximité institutionnelle, un programme est perçu comme une réussite si l'appareil se vend. Il faut voir là un basculement des représentations et des habitudes de comportements : la technologie ne doit être utilisée que si elle confère au produit un avantage commercial, et non plus pour elle-même. Toutes les améliorations ont pour objectif d'aboutir à une réduction sensible pour les compagnies aériennes des coûts d'achats, d'exploitation et de maintenance. Dès lors, ce sont les compagnies aériennes qui fixent les caractéristiques et le prix de l'appareil, alors qu'habituellement les Etats nationaux jouaient ce rôle de prescripteur. La famille des Airbus (programme lancé en 1967) est ainsi conçue en respectant cette logique : l'Airbus doit être un appareil simple et peu cher, correspondant aux besoins du marché en termes de qualité, de performance, de délais de livraisons, de coûts d'exploitation, de maintenance et de prix. Au final, le désengagement progressif de l'Etat français de l'actionnariat des entreprises françaises souhaité par les partenaires européens, les coopérations multipartites européennes engagées dès les années soixante et la spécialisation des sites qui en découlent, ouvrent la voie à l'intégration de l'industrie aéronautique européenne. EADS, comme forme de proximité organisationnelle, est l'expression concrète de la logique de marché : pour les acteurs de cette industrie, le principe d'efficacité économique doit maintenant prévaloir sur la préservation des intérêts strictement nationaux ce qui suppose la création d'une firme intégrée. Mais cette affirmation de la logique de marché ne signifie pas que la logique d'arsenal soit totalement occultée. La structure de pouvoir d'EADS démontre par certains aspects (Etats actionnaires, structure bicéphale) la survivance de cette dernière, la préservation des intérêts nationaux prévalant ponctuellement. La structure organisationnelle est bien le reflet d'une proximité institutionnelle composée de deux logiques, l'une dominant l'autre.

\subsubsection{Une proximité organisationnelle polymorphe déformée par les} rapports de pouvoir

EADS a été construit tout naturellement autour de l'ex GIE Airbus et ce pour plusieurs raisons. Le GIE fut la forme de coopération industrielle et commerciale la plus aboutie en Europe. Aerospatiale, Dasa et Casa ont ainsi appris à travailler ensemble depuis une trentaine d'année au fil des programmes Airbus : l'intégration n'en était que plus facile. Les chances de réussite de la fusion étaient encore accentuées par le fait que les Etats espagnol, allemand et français ont principalement apporté à la société unique européenne des actifs civils, par nature plus aisément mutualisables 
que les activités militaires. De fait, quel que soit le critère choisi, l'activité issue des programmes Airbus apparaît comme dominante au sein du groupe. En 2004 en effet, $48 \%$ des effectifs appartiennent à la Division Airbus, qui réalise $60 \%$ du chiffre d'affaires total et 57 $\%$ des prises de commandes tout en constituant la principale source de profits $\left(44 \%\right.$ de l'EBIT $\left.^{10}\right)$.

Sur le plan de la coordination politique, ce déséquilibre pose des difficultés internes tant le groupe est dépendant de la Division Airbus qui dispose de fait d'une large autonomie décisionnelle. Notamment des conflits se sont fait jour en ce qui concerne la définition de la stratégie d'Airbus, la maison mère ayant par exemple des difficultés à imposer ses vues à l'avionneur en terme de localisation des unités de production, la première envisageant une relocalisation sur le territoire américain refusée par l'équipe dirigeante de la Division Airbus.

Une autre conséquence de cette domination des activités de la Division Airbus dans EADS est le rejet en périphérie de certaines unités moins bien intégrées. Prenons le cas de l'une des unités bordelaises du groupe. Créé en juin 2003 pour favoriser l'intégration des activités française (EADS Launch Vehicules) et allemande (Astrium Space Infrastructure), EADS Space Transportation est un maître d'œuvre spécialisé dans le transport et les infrastructures spatiales. Si le groupe consolidé EADS ST voit sa charge d'activité se répartir équitablement autour des domaines de la Défense, des infrastructures orbitales et d'Ariane, le site de Bordeaux demeure fortement spécialisé dans le domaine militaire $(85 \%$ du niveau d'activité de l'établissement). Le site girondin n'est donc pas représentatif d'un groupe centré sur les activités civiles, d'autant que, dans le strict respect de la logique d'arsenal, son rôle clé dans la construction de la force de dissuasion française le place de facto sous le contrôle direct de l'Etat français. Si on le compare à ses homologues toulousains (Airbus), l'établissement est doté d'un statut périphérique au sein du groupe. La proximité organisationnelle, loin d'être isomorphe, présente ici des caractéristiques différentes selon les unités concernées. Elle se déforme en fonction des rapports de pouvoir, provenant des asymétries de ressources cognitives et matérielles, qui se diffusent dans une structure organisationnelle complexe. Une façon de réduire cette complexité, en attendant une intégration plus poussée des unités, est d'intensifier la coordination cognitive, l'autre facette de la proximité organisationnelle.

10 Earnings Before Interest and Taxes ou bénéfice opérationnel. 


\subsection{La proximité organisationnelle au sein d'EADS: la coordination cognitive}

Groupe récemment créé, EADS doit surmonter deux obstacles dans la coordination cognitive : gérer une multiplicité de sites porteurs de compétences qui se chevauchent, voire se dupliquent; favoriser la diffusion des routines entre des unités de cultures et de passés différents. La réponse est double : poursuivre la spécialisation des sites entamée durant les décennies précédentes par Airbus et créer un réseau interne de circulation d'information et des résultats de la recherche.

2.2.1. Une spécialisation croissante des sites d'EADS:
interpénétration des dimensions cognitive et politique de la
coordination

De façon générale, le groupe EADS accroît la division cognitive du travail entre ses différents établissements, suivant en cela le mouvement de spécialisation des sites initié en son temps par le GIE Airbus. Ce processus s'est accru au cours des années quatrevingt. Ainsi, à partir de 1988, chaque usine du groupe Aerospatiale se voit confier une partie homogène des éléments attribués à l'avionneur français par le GIE. Une double spécialisation produits/compétences est élaborée, évitant de la sorte la duplication des moyens industriels, améliorant le taux d'utilisation des machines et des compétences spécifiques, et diminuant les déplacements entre les usines (Kechidi, 1996). Par la suite, ce mouvement de spécialisation s'est encore accentué (Jalabert, Leriche et Zuliani, 2002). Des équipes intégrées Airbus sont désignées comme responsables de fonctions globales entières (voilures, tronçon, commandes de bord, mâts réacteurs, pointe avant, etc.), alors qu'auparavant diverses équipes dispersées en Europe pouvaient avoir une part de responsabilité dans la fonction. Toutefois, cette spécialisation n'est pas parfaitement transversale aux programmes, indiquant que le processus n'est qu'entamé. En effet, selon le type d'avion produit, la répartition des tâches entre les sites peut varier : ainsi, les A320 sont assemblés à Toulouse, tandis que les autres appareils de la famille (A318, A319, A321) le sont à Hambourg. Il faut voir dans cette duplication organisationnelle des sites d'assemblage une résurgence de la logique d'arsenal, le partenaire allemand souhaitant obtenir la tâche très symbolique de l'assemblage final d'une partie des Airbus pour des raisons notamment de prestige national. Ici la coordination politique, qui laisse une place aux intérêts nationaux relevant d'une logique d'arsenal, vient interférer avec les aspects plus cognitifs visant tout à la fois à rationaliser la distribution spatiale des compétences et leur 
diffusion intragroupe. La recherche de la satisfaction des intérêts purement nationaux fait alors l'objet d'un compromis (entre Français et Allemands dans notre illustration) d'ordre politique, au détriment de la performance économique. Il faut voir là une illustration de la perméabilité de la frontière analytique que nous avons tracée entre coordinations politique et cognitive pour donner corps à la proximité organisationnelle.

\subsubsection{La R\&D : fertilisation croisée et autonomie des unités}

Actuellement, les programmes de R\&D d'EADS sont en cours d'intégration. Ces activités sont fondées sur trois règles internes émises par la tête de groupe, la première renvoyant plus à une coordination d'ordre politique, les deux suivantes à une coordination cognitive : accorder à chaque Division la responsabilité du développement de ses produits afin de rester proche des besoins de la clientèle et laisser une grande autonomie dans la conception des programmes de recherches; coordonner les activités de recherche au sein du groupe par l'intermédiaire d'un réseau de recherche et de technologie (le "Réseau R\&T») permettant la circulation horizontale d'informations et des compétences internes, ce qui suppose la construction d'outils ; favoriser la centralisation des ressources, des compétences et des moyens de recherche par la création d'un centre commun de recherche (le CCR) réparti sur trois sites (Suresnes en France, Ottobrunn en Allemagne, Getafe en Espagne). L'objectif est d'inciter les diverses entités d'EADS à se tourner vers le CCR afin de participer à des programmes. Là encore, coordination cognitive et politique s'entremêlent. Sur le plan cognitif, cette organisation matricielle de la recherche favorise les fertilisations croisées entre les unités nouvellement intégrées dans EADS ${ }^{11}$. Sur le plan politique, la recherche amont rempli ici un rôle fédérateur (CCR), contrebalançant l'autonomie des unités à échanger par elles-mêmes (Réseau R\&T).

\subsection{Les proximités dans les relations verticales}

La logique de marché qui prévaut en interne au sein d'EADS est étendue aux relations verticales. Cette proximité institutionnelle, fondée sur la prédominance de la notion de coût, prend en effet progressivement place dans les relations de sous-traitance. Elle prend corps lorsque l'on analyse la proximité organisationnelle qui lie donneurs et preneurs d'ordres.

11 A l'exemple de la technologie de placement de fibres développée par EADS Casa pour des applications spatiales et qui connaît un débouché pour la fabrication du cône arrière de l'A380. 
Dans une logique d'arsenal, ces relations sont décrites sous la forme d'un réseau captif au sens où autour des grands donneurs d'ordres gravite un réseau d'entreprises sous-traitantes. L'avionneur, point focal du flux d'approvisionnement, l'est également au niveau de la conception. Il assure en interne la définition globale de l'avion et la conception détaillée des sous-ensembles qui le composent. Une nouvelle étape s'est faite jour autour des grands programmes sous le jeu d'au moins deux facteurs. D'une part, on note une profonde évolution de l'objet avion qui se complexifie. La composante industrielle de l'avion (la cellule) voit son importance, quantitative et qualitative, diminuer au profit des systèmes (Kechidi, 2005). Un avionneur ne peut dès lors maîtriser l'ensemble des systèmes compte tenu du spectre de compétences non similaires qu'il devrait acquérir. Aussi tend-il à se recentrer sur ses compétences foncières et à se borner dans un rôle d'architecte, conservant l'industrialisation des pièces de structure. D'autre part, afin de faire face à des dépenses de $R \& D$ toujours croissantes dans un contexte de plafonnement du potentiel des ressources traditionnelles, les constructeurs recherchent des économies en coût et en temps dès la phase de développement du produit jusqu'au service après-vente (Haas, Larré, Ourtau, 2001): la mobilisation de l'ensemble des ressources disponibles, en particulier celles présentes chez les fournisseurs, est à l'ordre du jour.

2.3.1. La coordination politique dans la proximité organisationnelle: structuration hiérarchique des fournisseurs

Que ce soit chez Airbus ou chez les autres constructeurs aéronautiques, on assiste à une recomposition de la pyramide des approvisionnements dans le respect des règles suivantes (Frigant et Talbot, 2005) : une réduction drastique du nombre de fournisseurs directs, qui se voient confier la charge complète de la conception et de la production des modules dont ils obtiennent la responsabilité ; la généralisation des pratiques de risk-sharing. Désormais, accéder directement à l'avionneur exige de participer au financement des investissements de R\&D et d'assumer ces responsabilités jusqu'à la certification de l'appareil. En outre, les investissements immatériels et matériels seront amortis sur un nombre précis d'avions l'équipementier obtenant l'exclusivité de la charge de travail - et ce n'est qu'au-delà de ce chiffre qu'il peut espérer réaliser un profit.

Le réseau de sous-traitance d'Airbus se présente comme une pyramide, véritable structure hiérarchique, organisée sur quatre niveaux (Kechidi, 2005) :

1- Les systémiers ou intégrateur de modules sont des entreprises qui participent à la conception et à la réalisation 
d'un sous-ensemble technique dont elles ont la responsabilité. Un systémier partage le risque financier avec l'avionneur en finançant sa R\&D et les coûts d'industrialisation ;

2- Les équipementiers fournissent soit un module technique autonome soit un module devant faire partie d'un ensemble technique plus complexe. Cette fourniture est soit faite sur la base d'un cahier des charges précis soit totalement confiée en étude et réalisation à l'équipementier ;

3- Les sous-traitants de spécialité sont des entreprises qui possèdent des actifs spécifiques (difficilement transférables) dans un domaine particulier ;

4- Les sous-traitants de capacité ou de production sont généralement de plus petites entreprises qui sont sélectionnées sur la base de leurs offres financières. Elles fournissent des pièces de production ou des services génériques largement standardisés et relevant de marchés très concurrentiels.

Dans cette structure de pouvoir, le systémier, et dans une moindre mesure l'équipementier, se voient dotés d'une certaine autonomie technique et industrielle, notamment dans les modes opératoires, afin d'atteindre un résultat imposé. Pour l'avionneur, coordonnateur politique, il s'agit d'externaliser des systèmes qu'il recevra fabriqués, protégés, contrôlés, bref prêts à monter.

2.3.2. La coordination cognitive dans la proximité organisationnelle: création de dispositifs collectifs de coordination

Le mouvement d'externalisation que nous venons d'évoquer, et la décomposition des compétences qu'il induit, supposent la construction de dispositifs collectifs de coordination élaborés, permettant à l'avionneur de conserver la maîtrise cognitive de la relation tout au long du processus de conception et de production. Il s'agit, par une harmonisation des outils de conception (matériels et logiciels de CFAO) et par la mise en place d'une ingénierie simultanée, de permettre aux sites européens d'échanger progressivement des données et d'homogénéiser les méthodes de formation, de support et de documentation. Concrètement, on assiste à une mise en liaison informatique (EDI) des différents sites des avionneurs et de leurs principaux fournisseurs. Cette coordination cognitive est complété par la constitution d'équipes plateaux en conception qui suppose une proximité géographique entre donneurs et preneurs d'ordres de premier rang. Autrement dit, le face à face, tout particulièrement dans les phases de conception commune, demeure essentiel. L'existence durable d'une telle proximité 
géographique entre preneurs et donneurs d'ordres est parfaitement illustrée par le cas toulousain ${ }^{12}$.

2.3.3. La proximité géographique dans les relations verticales : un rôle essentiel mais temporaire dans la coordination cognitive

Selon l'INSEE (2005), l'agglomération toulousaine concentre en 2004 les trois quarts des effectifs midi-pyrénéens des établissements liés au secteur aéronautique et spatial, les deux tiers des établissements liés ${ }^{13}$ à l'aéronautique de la Région. En outre, la plupart des prestataires de services et la quasi-totalité des établissements travaillant dans l'informatique sont situés dans l'aire urbaine toulousaine.

Il existe une première raison historique à l'existence d'un tissu ancien de systémiers, d'équipementiers et de PME soustraitantes autour de l'agglomération toulousaine. Dans le cadre d'une logique d'arsenal, l'Etat faisait appliquer sa politique d'aménagement du territoire par les industriels comme Aerospatiale, groupe public : ce dernier se voyait attribuer un rôle d'animateur de l'industrie locale, dans le sens où la charge de travail qu'il déléguait constituait une ressource matérielle attribuée par l'Etat qu'il fallait partager. Peu à peu, aux côtés des premières entreprises présentes de longue date dans la région ${ }^{14}$, s'est ajouté un ensemble de preneurs d'ordres captant la ressource publique. Depuis les années quatre-vingt le désengagement progressif de l'Etat du secteur aéronautique civil autorise une émancipation des industriels. Ce retrait étatique permet à EADS-Airbus, désormais en charge des approvisionnements, d'élargir la recherche des sous-traitants aux niveaux national et international, abandonnant son rôle de soutien public au tissu industriel local que lui imposait la logique d'arsenal. Par conséquent, si cette raison historique explique les localisations déjà anciennes, elle ne permet pas en revanche de rendre compte des nouvelles implantations constatées lors du développement du programme A380. Divers systémiers et équipementiers se sont en effet implantés à proximité des unités de décision, de R\&D et d'assemblage d'Airbus. Trois autres explications au moins, largement complémentaires les unes des autres, peuvent être fournies (Frigant et Talbot, 2003).

12 Selon Zuliani et Jalabert (2005), il existe une concentration similaire d'équipementiers aéronautiques autour de Madrid en raison de la présence d'EADS-Casa, d'Hambourg et des sites d'assemblages de l'A320 ou encore Bristol-Filton où se localisent des unités de production d'Airbus UK.

13 Par "lié", l'INSEE entend les emplois qui dépendent en partie des commandes du secteur.

14 Latécoère s'est implanté à Toulouse en 1917 suivi quelques années plus tard de Ratier à Figeac, de Messier à Pau, de Morane Saulnier à Tarbes.

174 Economie et Institutions $-n^{\circ} 6$ et $7-1^{\text {er }}$ et $2^{\text {e }}$ semestres 2005 
Sur un plan logistique, les livraisons effectuées par les systémiers et les équipementiers fournisseurs d'aérostructures se heurtent à la taille importante des composants. L'approvisionnement du site toulousain d'assemblage de l'A380 par des éléments réalisés notamment en Espagne (empennage horizontal), en Grande-Bretagne (voilure), à Hambourg (fuselage) ou encore à Saint-Nazaire (fuselage) oblige à construire des navires spéciaux (liaison maritime) puis une voie à grand gabarit entre Bordeaux et Toulouse (liaison routière), illustrant par là même les surcoûts qui proviennent d'un approvisionnement géographiquement éloigné. $\mathrm{Si}$ au sein des différentes composantes du groupe Airbus ce surcoût est accepté, il est moins évident que l'avionneur le tolère chez ses équipementiers au moment même où il accroît sa pression pour réduire ses coûts d'approvisionnement.

Sur un plan politique, l'enjeu économique du programme A380 et l'affirmation de Toulouse comme capitale industrielle de l'avionneur justifie d'un choix stratégique des équipementiers. La réalisation d'investissements à proximité constitue une preuve d'engagement dans la relation. Et cela que l'on se place du point de vue des équipementiers ou de l'avionneur. Côté équipementier, réaliser des investissements à proximité constitue un signal prouvant la volonté de s'impliquer à long terme auprès du constructeur toulousain, en particulier pour les industriels qui s'engagent pour la première fois de façon majeure auprès d'Airbus. C'est notamment le cas pour les équipementiers américains traditionnellement plus attachés au concurrent Boeing. Côté avionneur, l'efficacité induite par l'existence de ces investissements spécifiques localisés incite les donneurs d'ordres à octroyer aux preneurs d'ordres locaux le développement puis la réalisation industrielle des modules. Ces investissements constituent en quelque sorte un moyen de renforcer la relation bilatérale.

Sur un plan cognitif, un troisième ensemble de motifs renvoie aux méthodes de fabrication et au contenu du produit et justifie la réalisation d'unité de production à proximité. Nous distinguons les activités proprement industrielles des services. Du côté des activités industrielles, et concernant les méthodes de fabrication, la décomposition des objets techniques en sous-ensembles induit une forte densification des interactions nécessaires à la recomposition et à l'assemblage de l'avion (Kechidi, 2005). A cette décomposition accrue s'ajoute une complexité croissante du développement du produit, du fait de la nature novatrice de l'A380, ce qui incite de nombreux systémiers et équipementiers sélectionnés à implanter dans la zone toulousaine des équipes de recherche chargées de travailler en étroite collaboration avec les ingénieurs de l'avionneur. Ici, la proximité organisationnelle fondée sur l'ingénierie simultanée doit être complétée par de fréquents face à face. La proximité 
géographique atteint une phase plus intense lors de la mise en place d'équipes projets mixtes entre avionneurs, systémiers et équipementiers. C'est à l'occasion du développement de l'A340500/600 en 1997 qu'Airbus a développé sa première équipe plateau. L'A380 approfondit cette organisation avec la construction d'un plateau d'un millier d'ingénieurs et de techniciens à Toulouse où sont réunis les personnels de l'avionneur et des équipementiers. Ce besoin intense de face à face auquel répond l'équipe plateau concerne uniquement les phases de conception générale de l'avion et de développement pour mettre au point les solutions techniques retenues. Le plateau est ensuite dissout. Durant cette période de quelques mois, il permet de recombiner des compétences dispersées entre différents acteurs éloignés physiquement en vue d'innover et de résoudre un problème productif. La proximité géographique, ici transitoire, et plus précisément le face à face qu'elle autorise, facilite grandement cette recombinaison en permettant le transfert de connaissances, tout en réduisant l'incertitude issue de la dimension tacite inhérente à tous savoirs et les risques d'opportunisme (Boschma, 2004 ; cf. en outre l'article du même auteur dans ce numéro). Du côté des études et des services cette fois, il apparaît que la branche de la construction aéronautique fait de plus en plus appel à des sociétés orientées vers ce type d'activité au détriment des entreprises de construction mécanique. Selon les diverses enquêtes INSEE, les activités tertiaires passent ainsi de moins de $20 \%$ du total des emplois liés à la branche en 1982 à près de $45 \%$ en 1999 et $55 \%$ en 2002. Pour Zuliani et Jalabert (2005), la mise au point et l'utilisation de systèmes informatiques de plus en plus complexes rend obligatoire un contact entre les salariés d'Airbus et des SSII effectuant des développements, des mises au point, des contrôles et de la maintenance. Cela favorise une concentration croissante des activités sur l'aire urbaine toulousaine, non pas tant du fait de relocalisations d'entreprises, mais parce que les nouvelles entreprises de services qui se créent s'implantent à Toulouse, à la recherche d'une proximité géographique étroite.

\section{Conclusion}

Après avoir justifié la conservation des trois dimensions de la proximité, nous avons défini ces différentes formes à travers une grille de lecture institutionnaliste insistant sur le double effet contrainte versus libération de l'action individuelle. En outre, nous avons cherché à valider l'hypothèse qu'une proximité organisationnelle pouvait se décrire en distinguant, de façon purement analytique, la coordination politique de la coordination cognitive. Cette frontière analytique s'est d'ailleurs avérée poreuse à l'épreuve de la réalité. Cette même réalité nous a d'ailleurs conduits 
à distinguer deux composantes de la proximité institutionnelle, la logique d'arsenal et la logique de marché. C'est l'occasion pour nous d'affirmer le caractère profondément dynamique des formes de proximités qui se recomposent continuellement, puisqu'elles renvoient à un partage d'objets, institution, organisation, espace, eux-mêmes en perpétuelle évolution. Enfin, l'exemple de la formation d'équipes plateaux au sein des usines Airbus à Toulouse nous permet de souligner le caractère cette fois complémentaire de ces formes évolutives. La proximité géographique est dans notre cas apparue comme un complément aux autres formes de proximité. Si diverses interfaces organisationnelles mobilisant les technologies de l'information et des communications permettent une coordination cognitive continue à distance, en raison de la complexité du produit elles doivent être ponctuellement complétées par de fréquentes rencontres physiques qui justifient une co-localisation, même transitoire. Au-delà des proximités organisationnelle et géographique, la conception commune, objectif des équipes plateaux, suppose une compréhension mutuelle et un apaisement transitoire des conflits, bref une proximité institutionnelle. Au final, la proximité géographique permet de favoriser le partage de connaissances, mais sans se substituer ici aux proximités institutionnelle et organisationnelle. Ces proximités, comprises comme évolutives et complémentaires, permettent alors de caractériser les différentes formes d'action collective.

\section{Références}

Bazzoli L. et V. Dutraive, (2002), "L'entreprise comme organisation et comme institution. Un regard à partir de l'institutionnalisme de J. R. Commons", Economie et Institutions, $\mathrm{n}^{\circ} 12^{\mathrm{e}}$ semestre, pp. 4-46.

Becue M., S. Cancel, Y. Dupuy, V. Frigant, J. P. Gilly, M. Kechidi et D. Talbot, (2005), EADS et les stratégies territoriales dans le SudOuest de la France, GRES IFReDE LEREPS, rapport national étude EETSE, initiative communautaire INTERREG III-B SUDOE, avril.

Boschma R., (2004), "Does geographical proximity favour innovation ?", Quatrième Journées de la Proximité, Proximité, réseaux et co-ordination, Marseille, juin.

Caron A. et A. Torre, (2004), "Quand la proximité est source de tensions et de conflits : le cas des conflits d'usage et de voisinage dans les campagnes françaises" Quatrième Journées de la Proximité, Proximité, réseaux et co-ordination, Marseille, juin.

Commons J. R., (1931), "Institutional Economics", American Economic Review, December, pp. 648-657.

Commons J. R., (1934), Institutional Economics. Its Place in Political Economy, New York, The MacMillan Compagny.

Corei T., (1995), L'économie institutionnaliste, les fondateurs, collection "Économie de poche", Paris, Economica. 
Dosi G., D. Teece D. et S. Winter, (1990), "Vers une reformulation des frontières de la grande entreprise : la notion de cohérence", Revue $d^{\prime}$ Economie Industrielle, $\mathrm{n}^{\circ} 51,1^{\text {er }}$ trimestre, pp. 238-254.

Dupuy C. et A. Burmeister, (éds), (2003), Entreprises et territoires, les nouveaux enjeux de la proximité, Paris, La Documentation Française.

Dupuy C. et J. P. Gilly, (1996), "Apprentissage organisationnel et dynamique territoriale", dans Pecqueur B. (éd.), Dynamiques territoriales et mutations économiques, Paris, L'Harmattan.

Frigant V. et D. Talbot, (2003), "Convergence et diversité du passage à la production modulaire dans l'aéronautique et l'automobile en Europe", Journée de travail du GERPISA, MSH-EHESS, Paris, 7 février.

Frigant V. et D. Talbot, (2005), "Technological determinism and modularity: lessons from a comparison between Aircraft and Auto Industries in Europe", Industry and Innovation, vol.12, $\mathrm{n}^{\circ} 3$, september, pp. 337-335.

Gilly J. P. et Y. Lung, (2004), "Proximités, secteurs, territoires", Quatrième Journées de la Proximité Proximité, réseaux et coordination, Marseille, juin.

Gilly J. P. et A. Torre (éds.), (2000), Dynamique de proximité, Paris, L'Harmattan.

Gislain J. J., (2002), "Causalité institutionnelle : la futurité chez J. R. Commons", Economie et Institutions, ${ }^{\circ} 1,2^{\mathrm{e}}$ semestre, pp. 47-66.

Gislain J. J., (2003), "L’institution des relations industrielles : le cadre analytique de J. R. Commons", Economie et Institutions, $\mathrm{n}^{\circ} 2$, $1^{\text {er }}$ semestre, pp. 11-60.

Grinberg M., (2001), "Un cheval est-il un cheval ? Les mots, les faits, le capitalisme et le droit", dans Cahiers d'économie politique, Lecture de John R. Commons, n40-41, Paris, L'Harmattan, pp. 177-192.

Grossetti M., (1997), "Proximité spatiale et proximité relationnelle", colloque Proximités et coordination économique, Creuset, Gate, Lyon, mai.

Grossetti M. et M. Filippi, (2004), "Proximité et relations interindividuelles", dans B. Pecqueur et J. B. Zimmermann (éds.), Economie de Proximités, Paris, Hermès, Lavoisier.

Haas J., Larré F., Ourtau M. (2001), "R\&D dans le secteur aéronautique et spatial : tensions liées à un contexte nouveau", Les notes $d u$ LIRHE, $\mathrm{n}^{\circ} 348$, Novembre.

Hodgson G., (2000), "What is the essence of institutional economics?" Journal of Economic Issues, vol. 34, n², Juny, pp. 317-329.

Insee, (2005), Aéronautique, espace et sous-traitance en MidiPyrénées. Résultats de l'enquête 2004, Toulouse, dossier $\mathrm{n}^{\circ} 125$.

Jalabert G., F. Leriche F. et J. M. Zuliani, (2002), Système productif, réseaux internationaux de villes, dynamiques urbaines: les villes européennes de l'aéronautique, CIEU, Université de Toulouse Le 
Mirail. Rapport de Recherche pour le Ministère de la Recherche et de la Technologie, action concertée Ville, novembre.

Jullien B., (2004), Pour une mésoéconomie politique. Eléments d'une approche institutionnaliste $d u$ changement dans les industries. Habilitation à diriger des recherches, Université Montesquieu Bordeaux IV, avril.

Kechidi M., (1996), "Coordination interentreprises et sous-traitance : le cas d'Aerospatiale", Revue d'Economie Régionale et Urbaine, $\mathrm{n}^{\circ} 1$, pp. 99-120.

Kechidi M., (2005), "Mutation des relations verticales dans l'industrie aérospatiale : une analyse de la relation de sous-traitance d'Airbus en Midi-Pyrénées", XLIème Colloque de l'ASRDFL Villes et Territoires face aux défis de la mondialisation, Dijon, septembre.

Kirat T. et C. Lefranc, (2004), "Les conflits d'usage et leur expression territoriale: une analyse des profils contentieux de sept départements français", Quatrième Journées de la Proximité, Proximité, réseaux et co-ordination, Marseille, juin.

Kirat T. et Y. Lung, (1995), "Innovations et proximités : le territoire, lieu de déploiement des processus d'apprentissage", dans N. Lazaric et J. M. Monnier (éds.), Coordination économique et apprentissage des firmes, Paris, Economica.

Laganier R., B. Villalba et B. Zuindeau, (2002), "Le développement durable face au territoire : éléments pour une recherche pluridisciplinaire", Revue Développement Durable et Territoires, dossier $\mathrm{n}^{\circ} 1$ Approches territoriales du Développement Durable.

Menard C., (1994), "La nature de l'innovation organisationnelle, éléments de réflexion", Revue d'Économie Industrielle, numéro hors série Économie industrielle : développements récents, juillet, pp. 173-192.

Muller P., (1988), Airbus l'ambition européenne, logique d'Etat, logique de marché, collection "Logiques Sociales", Paris, L'Harmattan.

Pecqueur B., (1997), "Processus cognitifs et construction des territoires économiques", dans B. Guilhon, P. Huard, M. Orillard et J. B. Zimmermann (éds.), Économie de la connaissance et des organisations, Paris, L'Harmattan.

Pecqueur B. et J. B. Zimmermann, (2004), "Introduction. Les fondements d'une économie de proximités" dans B. Pecqueur et J. B. Zimmermann J. B. (éds.), Economie de Proximités, Paris, Hermès, Lavoisier.

Rallet A. et A. Torre, (2004), "Proximité et localisation", Economie rurale, $\mathrm{n}^{\circ} 280$, mars avril, pp. 25-41.

Reynaud B., (2001), "Suivre des règles dans les organisations", Revue d'Economie Industrielle, 4e trimestre, pp. 53-68.

Talbot D., (2000), "Institutional dynamics on localised inter-firm: the case of Aerospatiale and the Toulousian subcontractors", European Urban \& Regional Studies, vol.3, July, pp. 223-236. 
Torre A. et M. Filippi, (éds.) (2005), Proximité et changements socioéconomiques dans les mondes ruraux, Paris, INRA Editions.

Vant A., (1998), "Proximités et géographies", dans M. Bellet, T. Kirat et C. Largeron (éds.), Approches multiformes de la proximité, Paris, Hermès.

Zuliani J.M. et G. Jalabert, (2005), "L'industrie aéronautique européenne: organisation industrielle et fonctionnement en réseaux", L’Espace géographique, n² 2, pp. 117-133.

180 Economie et Institutions $-n^{\circ} 6$ et $7-1^{\text {er }}$ et $2^{\text {e }}$ semestres 2005 